\title{
Does nebulized magnesium reduce the rate of hospitalization compared to placebo in pediatric patients with acute refractory asthma treated in the emergency department?
}

\author{
Dana Stewart $^{1} \mathbb{D} \cdot$ Meaghan Mackenzie $^{1} \cdot$ Shawn Dowling ${ }^{1}$
}

Received: 14 January 2021 / Accepted: 23 March 2021 / Published online: 21 April 2021

(c) Crown 2021

Keywords Pediatric $\cdot$ Asthma $\cdot$ Magnesium

Full Citation: Schuh S et al. Effect of nebulized magnesium vs placebo added to albuterol on hospitalization among children with refractory acute asthma treated in the emergency department: a randomized clinical trial. JAMA 2020 Nov 24; 324(20): 2038-2047.

Abstract Link: https://pubmed.ncbi.nlm.nih.gov/33231 $663 /$

Article Type: Randomized clinical trial, Therapy

Ratings:Methods_-5/5, Usefulness-4/5

\section{Introduction}

\section{Background}

Nebulized magnesium provides a less invasive means of delivering therapy in pediatric asthma exacerbations. However, evidence to support its use is lacking.

\section{Objectives}

To determine whether nebulized magnesium reduces hospitalization rates compared to placebo in pediatric patients with refractory acute asthma in the emergency department (ED).

Dana Stewart

dana.stewart2@ucalgary.ca

1 University of Calgary, 2500 University Dr NW, Calgary, AB T2N 1N4, Canada

\section{Methods}

\section{Design}

Randomized, double-blind, parallel-group, and placebocontrolled trial.

\section{Setting}

Seven tertiary-care pediatric emergency departments in Canada.

\section{Subjects}

Healthy children aged 2-17 years with moderate to severe asthma, defined as a Pediatric Respiratory Assessment Measure (PRAM) score $\geq 5$ after $1 \mathrm{~h}$ of therapy with an oral corticosteroid and three treatments of inhaled albuterol and ipratropium.

\section{Intervention}

Randomized to three nebulized albuterol treatments with either magnesium sulfate or saline placebo.

\section{Outcomes}

Primary outcome was hospitalization for asthma within $24 \mathrm{~h}$. Secondary outcomes included PRAM score, respiratory rate, oxygen saturations, blood pressure, and albuterol treatments within $240 \mathrm{~min}$. 
Table 1 Secondary outcomes

\begin{tabular}{lc}
\hline Outcome & Adjusted risk difference $(95 \%$ CI $)$ \\
\hline Additional albuterol treatments within $240 \mathrm{~min}$ & $0.94(0.78-1.14, P=0.53)$ \\
\hline Outcome (changes from baseline to $240 \mathrm{~min})$ & Adjusted difference-in-difference $(95 \%$ CI) \\
\hline PRAM score & $0.14(-0.23-0.51, P=0.45)$ \\
Respiratory rate (breaths/minute) & $0.31(-1.17-1.79, P=0.68)$ \\
Oxygen saturation $(\%)$ & $-0.05(-0.54-0.45 \%, P=0.86)$ \\
Systolic blood pressure $(\mathrm{mmHg})$ & $0.61(-1.64-2.85, P=0.60)$ \\
\hline
\end{tabular}

\section{Main results}

5846 patients were screened for inclusion in the study and 4332 were excluded, resulting in a total of 816 enrolled patients (409 received magnesium, 407 received placebo). Of the patients randomized to the magnesium treatment group, $178 / 409(43.5 \%)$ were hospitalized, compared to $194 / 407(47.7 \%)$ in the placebo group (difference $-4.2 \%$; absolute risk difference $95 \% \mathrm{CI}-11.0-2.8 \% ; P=0.26)$. Furthermore, subgroup analyses demonstrated no statistically significant treatment effect for the primary outcome Table 1.

\section{Appraisal}

\section{Strengths}

- Clinically relevant question with limited high-quality evidence available prior to this study

- Patient centered primary outcome

- Standardized and optimal initial therapy

- Baseline demographic and prognostic factors similar between study groups

- Stratified for potential covariates (age and site)

- Methodologically sound study protocol

- Large magnesium dose delivered by an efficient nebulizer

- Multiple ED's included: high external validity

\section{Limitations}

- No standardized criteria for hospitalization, decision left up to physician judgement.

- Only a small number of included patients had severe respiratory distress, as critically ill patients were excluded from the study. Furthermore, no definition of critically ill was provided by the authors.

- Study potentially underpowered to detect small differences in favour of nebulized magnesium.

- However, the clinical significance of these smaller differences is debatable.

\section{Context}

The British Thoracic Society Intercollegiate Guidelines Network recommends considering inhaled magnesium in the first hour of therapy for children with acute severe asthma [1]. This recommendation came from a multicenter EDbased trial of 508 children in the United Kingdom. Powell et al. demonstrated that children who received inhaled magnesium resulted in statistically significant improvement in the Yung asthma score [2]. However, the difference was not clinically significant. Alternatively, Alansari et al. completed a single-center study that demonstrated that nebulized magnesium did not reduce time to discharge in 365 hospitalized patients [3]. The Canadian Thoracic Society does not recommend for or against nebulized magnesium.

\section{Bottom line}

In this large, multicenter, and randomized clinical trial, nebulized magnesium showed no benefit compared to placebo in pediatric patients with acute refractory asthma exacerbations presenting to the ED. We do not recommend the use of nebulized magnesium in this setting.

\section{Declaration}

Conflict of interest The author's have no conflicts of interest to declare.

\section{References}

1. Scottish Intercollegiate Guidelines Network. British guideline on the management of asthma. British guideline on the management of asthma. Thorax. 2014;69:1-192.

2. Powell C, Kolamunnage-Dona R, Lowe J, et al. MAGNETIC study group.Magnesium sulphate in acute severe asthma in children (MAGNETIC): a randomised, placebo-controlled trial. Lancet Respir Med. 2013;1(4):301-8. https://doi.org/10.1016/S22132600(13)70037-7.

3. Alansari K, Ahmed W, Davidson BL, Alamri M, Zakaria I, Alrifaai M. Nebulized magnesium for moderate and severe pediatric asthma: a randomized trial. PediatrPulmonol. 2015;50(12):11919. https://doi.org/10.1002/ppul.23158. 